\title{
The temporal power spectrum of atmospheric fluctuations due to water vapor
}

\author{
O.P. Lay \\ Division of Physics, Mathematics and Astronomy, California Institute of Technology, Pasadena CA 91125, U.S.A.
}

Received May 17; accepted August 2, 1996

\begin{abstract}
Irregular variations in the refractivity of the atmosphere cause fluctuations in the phase measured by interferometers, limiting the spatial resolution that can be obtained. For frequencies up to the far infrared, water vapor is the dominant cause of the variations. The temporal power spectrum of the phase fluctuations is needed to assess correction schemes such as phase referencing using a nearby calibrator and water vapor radiometry.
\end{abstract}

A model is developed for the temporal power spectrum of phase fluctuations measured by an interferometer through a layer of Kolmogorov turbulence of arbitrary thickness. It is found that both the orientation of the baseline with respect to the wind direction and the elevation of the observations can have a large effect on the temporal power spectrum. Plots of the spectral density distribution, where the area under the curve is proportional to phase power, show that substantial contributions from length scales as long as 100 times the interferometer baseline are possible.

The model is generally consistent with data from the $12-\mathrm{GHz}$ phase monitor at the Owens Valley Radio Observatory, and allows the data to be extrapolated to an arbitrary baseline, observing frequency and elevation. There is some evidence that there can be more than one component of turbulence present at a given time for the Owens Valley.

The validity of the frozen turbulence assumption and the geometrical optics approximation is discussed and found to be reasonable under most conditions. The models and data presented here form the basis of an analysis of phase calibration and water vapor radiometry (Lay 1997).

Key words: atmospheric effects - instrumentation: interferometers - site testing - techniques: interferometric

\section{Introduction}

The performance of radio interferometers, particularly those operating at millimeter and submillimeter wavelengths, is often limited by fluctuations in the refractive index of the earth's atmosphere caused by water vapor. There is currently an active effort to correct for these fluctuations by using the techniques of water vapor radiometry (e.g. Welch 1994; Bremer 1995) and fast switching between the target and calibrator objects (Holdaway 1992; Holdaway \& Owen 1995).

The refractivity of water vapor is dominated by contributions from strong lines in the far infrared part of the spectrum. The refractivity of water vapor is therefore almost constant from radio to submillimeter wavelengths, and is substantially lower in the optical, where temperature variations become the dominating factor. The broad distribution of water vapor in the atmosphere falls off with altitude and has a scale height of approximately $2 \mathrm{~km}$. The fluctuations, however, arise from an irregular distribution of water vapor generated by turbulent mixing.

A comprehensive treatment of the theory of wave propagation in random media is given by Tatarskii (1961, 1971). More recent developments can be found in Tatarskii et al. (1992). Treuhaft \& Lanyi (1987) made numerical integrations to model the effect of a turbulent layer of finite thickness, applying the results to VLBI observations. Most discussions of the theory of atmospheric turbulence concentrate on the structure function of the fluctuations, which describes how the phase difference between two points in space varies as a function of their separation. Simple theory (see next section) predicts that the structure function follows a power law, and there have been several measurements using radio interferometers to test this relationship (e.g. Armstrong \& Sramek 1982; Sramek 1990; Coulman \& Vernin 1991, all using the Very Large Array; Wright \& Welch 1990, using Berkeley-IllinoisMaryland Association Millimeter Array; Olmi \& Downes 1992, using the millimeter interferometer of the Institut de Radioastronomie Millimétrique). There is a wide scatter in the measured power law indices, some of which is 
due to the difficulties in measuring the phase fluctuations over sufficiently long time intervals (see Sect. 4.6).

An alternative approach is to use an instrument dedicated to observing atmospheric phase fluctuations; existing phase monitors are interferometers that observe a tone of $\sim 12 \mathrm{GHz}$ from a geosynchronous communications satellite. The phase monitor at the summit of Mauna Kea (Masson 1993) and at the Nobeyama Radio Observatory (Ishiguro et al. 1990) have been operating the longest. These have since been joined by similar instruments at the Owens Valley Radio Observatory (OVRO), the Very Long Baseline Array station on Mauna Kea, and two in Chile which are being used to conduct site tests for the National Radio Astronomy Observatory's proposed Millimeter Array and the Japanese Large Millimeter and Submillimeter Array.

This paper focuses on the temporal power spectrum of atmospheric phase fluctuations measured with the OVRO phase monitor. This analysis is particularly useful for assessing the timescales over which the fluctuations are important, and will be used as the basis for a companion paper that studies phase calibration schemes, both with and without correction from water vapor radiometry. The primary aim of this paper is to develop the tools needed to understand phase monitor power spectra; it is not to make a detailed investigation of the properties of the atmosphere.

The next section describes how simple theory is used to form a model of phase fluctuations which includes the effects of a finite thickness of the turbulent layer, the orientation of the baseline with respect to the wind, and the elevation of the observations. Following this is a brief description of the OVRO phase monitor, the data processing, and an analysis of an illustrative sample of data (Sect. 3). There is then a discussion of the issues and implications of this work, including how to extrapolate data measured by a phase monitor to interferometers with different baselines observing at arbitrary elevation.

\section{The model}

\subsection{Turbulence, structure functions and power spectra}

The simple model developed here will be used to interpret the data from the phase monitor presented in the next section. It is intended to emphasize the relationships between different quantities, rather than to be mathematically rigorous.

The inhomogenous distribution of water vapor in the atmosphere is the result of a turbulent velocity field acting on large scale concentrations of water vapor. Turbulence is injected into the atmosphere on large scales by processes such as convection, the passage of air past obstacles and wind shear, and cascades down to smaller scales where it is eventually dissipated by viscous friction. Between the outer scale of injection and the inner scale of dissipation- known as the inertial range - it is a good approximation to say that kinetic energy is conserved, and simple dimensional arguments predict that for 3-dimensional, isotropic turbulence, the power spectrum is described by a power law with an index of $-11 / 3$. This is the Kolmogorov Power Spectrum (Tatarskii 1961, 1971).

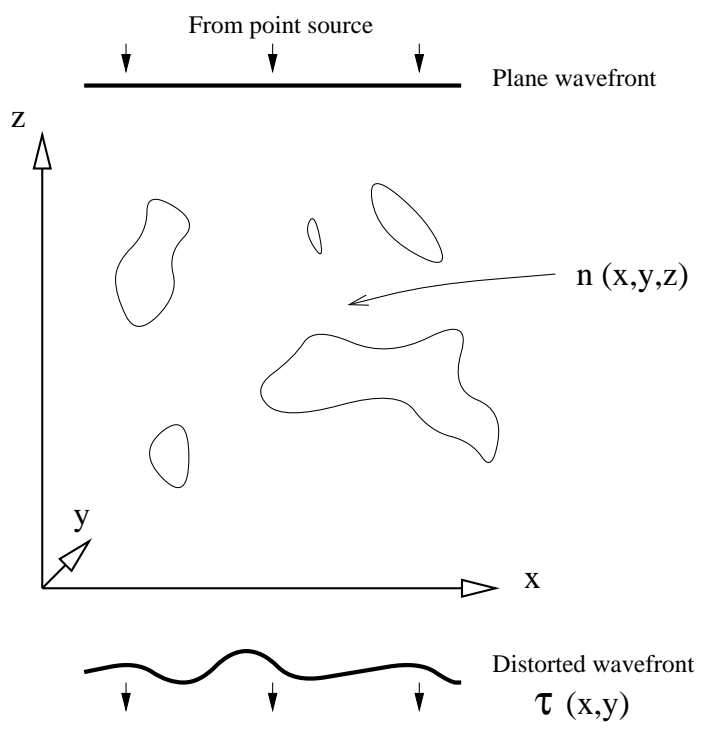

Fig. 1. A plane wavefront from a distant point source is distorted by variations in the refractivity $n$ as it passes through the atmosphere

Figure 1 shows how a plane wavefront from a distant point source is distorted as it passes through an atmosphere containing variations in the refractivity $n(x, y, z)$ (= refractive index -1$)$. Figure 2 illustrates the relationships between important quantities. The refractivity field $n(x, y, z)$ can be integrated along the line of sight (zaxis) to give the wavefront delay $\tau(x, y)$. These have 3$\mathrm{D}$ and 2-D Fourier Transforms given by $\widetilde{n}\left(q_{x}, q_{y}, q_{z}\right)$ and $\widetilde{\tau}\left(q_{x}, q_{y}\right)$, respectively, where $q$ denotes a spatial frequency. The Fourier Transforms are implicitly performed over a finite volume containing the scales of interest in the $x-y-z$ domain, ensuring that the integrals remain finite. The corresponding power spectra are $P_{n}$ and $P_{\tau}$. The autocorrelation functions for $n(x, y, z)$ and $\tau(x, y)$ are $A_{n}\left(\boldsymbol{R}_{x y z}\right)$ and $A_{\tau}\left(\boldsymbol{R}_{x y}\right)$, which in turn are related to the $3-\mathrm{D}$ and 2-D structure functions, respectively, of the refractivity field. For the case of fully three-dimensional Kolmogorov turbulence, the 2-D structure function $D_{\tau}\left(\boldsymbol{R}_{x y}\right)$ that gives the variance of the delay difference between two lines of sight separated by $\boldsymbol{R}_{x y}$, is proportional to $R_{x y}^{5 / 3}$. The details of the calculations can be found in the literature (e.g. Tatarskii 1961, 1971; Thompson et al. 1986). An implicit assumption is that the wavefront delay at a given location depends only on the refractivity field along the line 


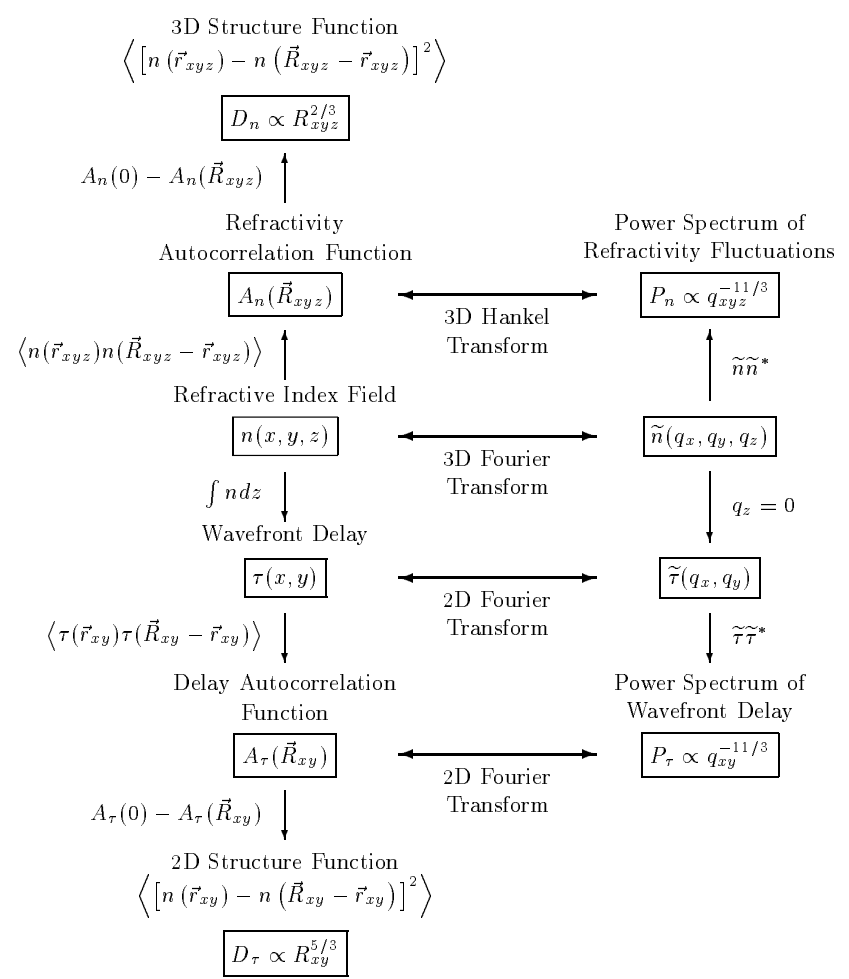

Fig. 2. Relationships between the refractivity field $n(x, y, z)$, wavefront delay, structure functions and power spectra. Power law indices, where given, are appropriate for a very thick layer of Kolmogorov turbulence. Variables: $(x, y, z)$ are spatial coordinates, $\left(q_{x}, q_{y}, q_{z}\right)$ are the corresponding spatial frequencies; $\boldsymbol{r}_{x y z}$ and $\boldsymbol{R}_{x y z}$ are a position and displacement in the 3-D space, respectively; $\boldsymbol{r}_{x y}$ and $\boldsymbol{R}_{x y}$ are their equivalents in the $x-y$ plane; $\boldsymbol{q}_{x y z}$ and $\boldsymbol{q}_{x y}$ are 3 -D and 2-D spatial frequencies: $q_{x y z}=\left(q_{x}^{2}+q_{y}^{2}+q_{z}^{2}\right)^{1 / 2} ; q_{x y}=\left(q_{x}^{2}+q_{y}^{2}\right)^{1 / 2}$; $r_{x y z}=\left(x^{2}+y^{2}+z^{2}\right)^{1 / 2} ; r_{x y}=\left(x^{2}+y^{2}\right)^{1 / 2}$

of sight; this is the geometrical optics approximation and is discussed in Sect. 4.

The emphasis in the past has been on the 2-D structure function $D_{\tau}\left(\boldsymbol{R}_{x y}\right)$. The focus of this analysis is the spatial power spectrum of the wavefront delay $P_{\tau}\left(q_{x}, q_{y}\right)$. In the next section the layer of turbulence is considered to be of effectively infinite thickness, so that the turbulence is isotropic; subsequent sections deal with layers of finite thickness.

\subsection{From refractivity to interferometer phase}

An interferometer, comprising a pair of antennas looking vertically up through the atmosphere separated by a baseline $\mathbf{d}=\left(d_{x}, d_{y}\right)$, is sensitive to the difference in the delay between the two signals received. This response is illustrated schematically in Fig. 3, where the two circles represent positive and negative delta functions at the location of the antennas. The Fourier Transform of this response is given by $2 \sin \left\{\pi\left(d_{x} q_{x}+d_{y} q_{y}\right)\right\}$, so that the
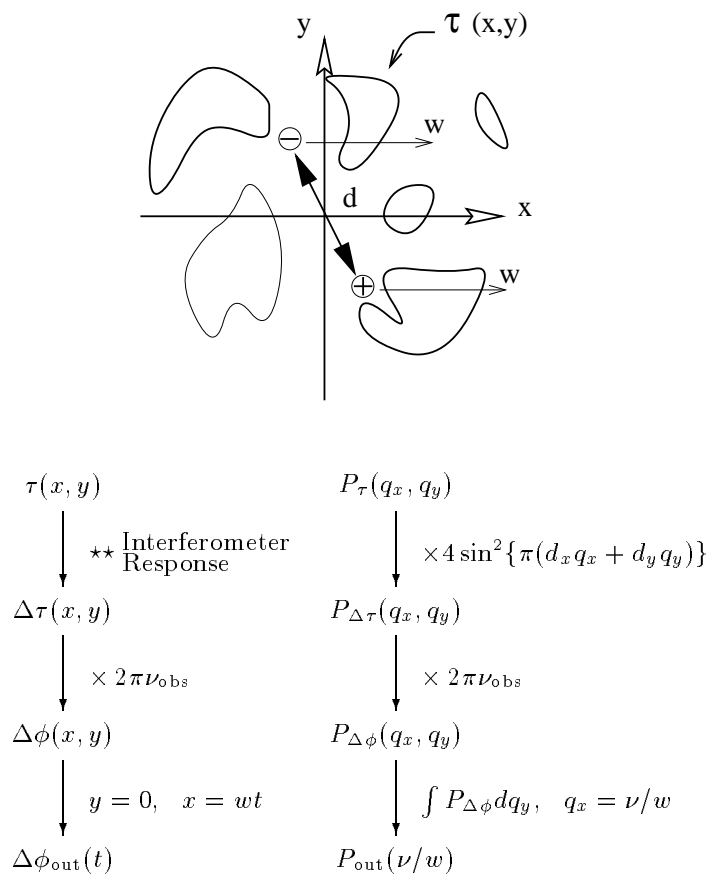

Fig. 3. The response of an interferometer with baseline $\mathbf{d}$ to a wavefront delay $\tau(x, y)$ moving at windspeed $\mathbf{w}$. On the left, $\tau(x, y)$ is mapped in stages onto the phase difference $\Delta \phi_{\text {out }}(t)$ measured by the interferometer as a function of time. The corresponding power spectra at each stage are shown on the right

interferometer acts as a spatial filter: the excess delay introduced by a fluctuation that is much larger than the baseline (i.e. $d_{x} q_{x}+d_{y} q_{y} \ll 1$ ) is very similar at each antenna and therefore gets canceled out to some extent. The power spectrum $P_{\Delta \tau}$ of this filtered signal is the product of the atmosphere's intrinsic power spectrum $P_{\tau}$ and the square of the filtering function:

$$
\begin{aligned}
P_{\Delta \tau} & =4 P_{\tau} \sin ^{2}\left\{\pi\left(d_{x} q_{x}+d_{y} q_{y}\right)\right\} \\
& \propto\left(q_{x}^{2}+q_{y}^{2}\right)^{-\frac{11}{6}} \sin ^{2}\left\{\pi\left(d_{x} q_{x}+d_{y} q_{y}\right)\right\}
\end{aligned}
$$

An interferometer actually measures the difference in the phase of the two signals received: $\Delta \phi(x, y)=$ $2 \pi \nu_{\mathrm{obs}} \Delta \tau(x, y)$ and $P_{\Delta \phi}=\left(2 \pi \nu_{\mathrm{obs}}\right)^{2} P_{\Delta \tau}$, where $\nu_{\mathrm{obs}}$ is the frequency of the radiation being observed.

Figures $4 \mathrm{a} \& \mathrm{~b}$ show contour plots of the quantity $P_{\Delta \phi} q_{x} q_{y}$ as a function of $\log q_{x}$ and $\log q_{y}$ for the cases of $\left(d_{x}=100 \mathrm{~m}, d_{y}=0\right)$ and $\left(d_{x}=0, d_{y}=100 \mathrm{~m}\right)$, respectively, i.e. wind along the baseline and wind perpendicular to the baseline. The spatial frequencies $q_{x}$ and $q_{y}$ have units $\mathrm{m}^{-1}$, such that $q=\lambda^{-1}$ for a disturbance with wavelength $\lambda$ (note: $\lambda$ is not the wavelength of the radiation here). The logarithmic axes (base 10) are necessary to cover the large range of scales and by plotting equally spaced contours of $P_{\Delta \phi} q_{x} q_{y}$ the volume under the 


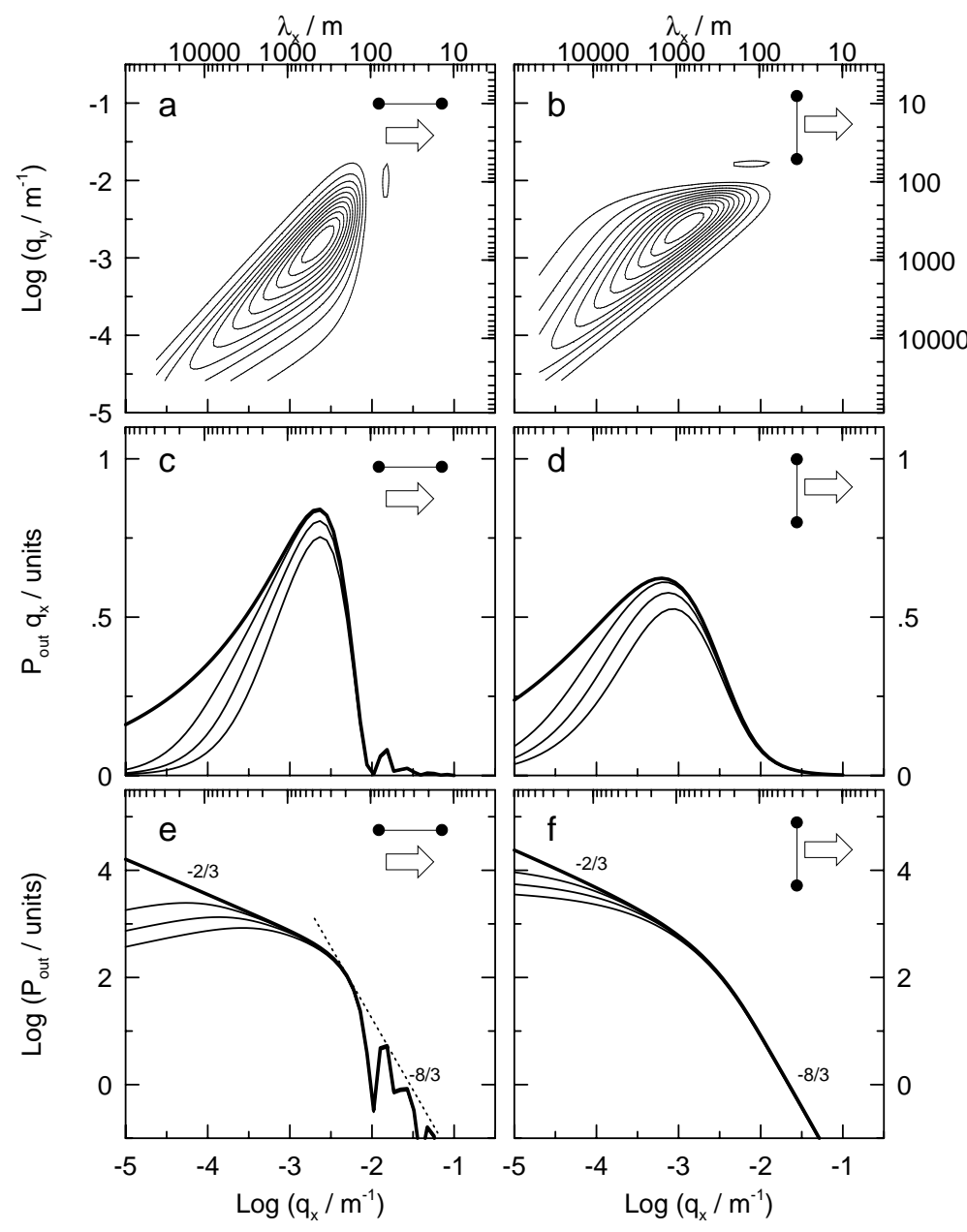

Fig. 4. a) Contour plot showing phase power as a function of spatial frequency $\left(q_{x}, q_{y}\right)$ for $\left(d_{x}=100 \mathrm{~m}, d_{y}=0\right)$ i.e. wind blowing parallel to the baseline, as indicated by the symbol. The contours are evenly spaced in the quantity $P_{\Delta \phi} q_{x} q_{y}$, so that volume under the contours is proportional to phase power. b) Contour plot for wind blowing perpendicular to baseline. The corresponding spatial scales are also shown. c \& d) Result of integrating the above functions over $\log q_{y}$. Area under the curves is proportional to phase power. The thick lines are for an unbounded turbulent region; the thin lines show the change in shape as the thickness of the turbulent layer $\Delta h$ is reduced to $5 \mathrm{~km}, 2 \mathrm{~km}$ and $1 \mathrm{~km}$, and have been normalised to have the same power on small scales. e \& f) Plots of the same curves on Log - Log axes. The asymptotic gradients for the unbounded case are shown. The corresponding frequency scale is obtained by the relation $\log \nu=\log q_{x}+\log w$. All Log scales are to base 10 contours is proportional to the variance $\Delta \phi$, i.e. the plot shows the contributions to the variance from different spatial frequencies. Although these plots are for $d=100 \mathrm{~m}$, the response for a longer (shorter) baseline is simply obtained by shifting the contours down and to the left (up and to the right). For example, for $d=200 \mathrm{~m}$ shift by $-0.3(=-\log 2)$ in both the $x$ and $y$ directions. It can be shown that the volume under the contours, that is the total variance in $\Delta \phi$, is proportional to $d^{5 / 3}$. This is the 2 -D phase structure function evaluated for a separation $d$ (Fig. 2).

The pattern of turbulence is blown at windspeed $w$ over the interferometer. Here it is assumed that $w$ is uniform in speed and direction over the volume containing the turbulence, and that the pattern of turbulence is essentially fixed over the time interval needed for the pattern to blow through a line of sight. This is the assumption of frozen turbulence and is addressed further in Sect. 5. It is convenient to consider the turbulence as fixed and the antennas as moving at speed $w$ in the $x$-direction, as shown in Fig. 3. $\Delta \phi(x, y)$ is sampled only along the $x$-axis, such that $x=w t$, where $t$ is time: $\Delta \phi_{\text {out }}(t)=\Delta \phi(w t, 0)$. The power spectrum $P_{\text {out }}(\nu)$ is derived by integrating $P_{\Delta \phi}$ over $q_{y}$. Here $\nu$ is the temporal frequency of phase variations in the output of the interferometer, not to be confused with the frequency of the observed radiation $\nu_{\text {obs }}$. For example, a fluctuation in the refractivity with a spatial periodicity of $200 \mathrm{~m}$ in the $x$-direction (i.e. $q_{x}=0.005 \mathrm{~m}^{-1}$, $\left.\log q_{x}=-2.3\right)$ gives rise to a measured phase fluctuation of period $40 \mathrm{~s}(\nu=0.0025 \mathrm{~Hz})$ if $w=5 \mathrm{~m} \mathrm{~s}^{-1} . P_{\text {out }} q_{x}$ is plotted against $\log q_{x}(=\log \nu-\log w)$ in Figs. 4c and d. Plotting $P_{\text {out }} q_{x}$ ensures that area under the curve is proportional to the variance of $\Delta \phi$, i.e. the curve represents a spectral density distribution.

The same models are plotted on Log - Log axes in Figs. 4e and f, illustrating the broken power law dependence of $P_{\text {out }}$ on $\nu$ : fluctuations much smaller than the baseline are uncorrelated between the two antennas and $P_{\text {out }} \propto \nu^{-8 / 3}$; fluctuations much larger than the baseline give $P_{\text {out }} \propto \nu^{-2 / 3}$.

\subsection{The effect of baseline orientation and length}

There are clear differences between the case shown in Figs. 4a, c and e where the wind blows along the baseline, and that shown in $b, d$ and $f$ where they are perpendicular. In the former case the contributions to the variance of $\Delta \phi$ are quite sharply peaked around scales corresponding 
to $\sim 5 d$, compared to a softer peak centered on scales of $\sim 15 d$ for the latter. The break in the power law is also more evident in Fig. 4e than in $\mathrm{f}$, and occurs at a spatial frequency given by $\log q_{x}=\log 2.5-\log (d / 100 \mathrm{~m})$. When the wind is neither parallel nor perpendicular to the baseline, the shapes of the curves are intermediate between the two extremes shown. The nulls in Figs. 4c and e are a direct result of the interferometer's response - the $\sin ^{2}\left(\pi d_{x} q_{x}\right)$ of Eq. (2) - and are suppressed when the wind is not blowing along the baseline.

The effect of the size of the individual antennas has been ignored up to this point; fluctuations much smaller than the effective aperture are smeared out, but since there is very little power on scales less than $d$, this approximation is justified. The curves plotted are for $100 \mathrm{~m}$ baselines. For a baseline length $d$, shift the the curves in $\mathrm{c}$ and $\mathrm{d}$ to the left by $\log (d / 100 \mathrm{~m})$; the vertical scale should also be increased by a factor $(d / 100 \mathrm{~m})^{5 / 3}$. The phase power is the same on small scales where there is no correlation between the two lines of sight and increases on large scales for the longer baselines.

\subsection{The finite thickness of the turbulent layer}

Until this point it has been assumed that the turbulent region is effectively infinite in all directions. The three other curves in Figs. 4c, d, e and f illustrate the effect on the phase power spectrum of an atmosphere with vertical thickness $\Delta h$ of $5 \mathrm{~km}, 2 \mathrm{~km}$ and $1 \mathrm{~km}$. In Fig. 2, the expressions for $\tau(x, y)$ and $P_{\tau}\left(q_{x}, q_{y}\right)$ are now given by

$$
\begin{aligned}
\tau(x, y) & =\int_{0}^{\Delta h} n \mathrm{~d} z, \\
P_{\tau}\left(q_{x}, q_{y}\right) & =\int_{-\infty}^{+\infty}|\widetilde{n}|^{2}(\Delta h)^{2} \operatorname{sinc}^{2}\left(\pi \Delta h q_{z}\right) \mathrm{d} q_{z} .
\end{aligned}
$$

The values of $P_{\text {out }}\left(q_{x}\right)$ are calculated by numerical integration. The curves have been normalized so that the power on small spatial scales is the same, to emphasize the differences in the shapes of the distributions. The finite value of $\Delta h$ takes effect for $q_{x} \lesssim \Delta h^{-1}$ : the distribution of phase power becomes narrower as there is less power on large spatial scales (or lower temporal frequency) and the $\log -\log$ plots deviate from the gradient of $-\frac{2}{3}$. The exact behavior depends on the orientation of the wind direction with respect to the baseline. The curves shown are for a $100 \mathrm{~m}$ baseline, but the shape of the curve is the same for a given value of $d / \Delta h$.

When the baseline is much longer than the thickness of the turbulent layer, the power law index of $-8 / 3$ on scales smaller than $\Delta h$ flattens to an index of $-5 / 3$ for larger scales (Fig. 5). There is further flattening when the baseline length is exceeded.

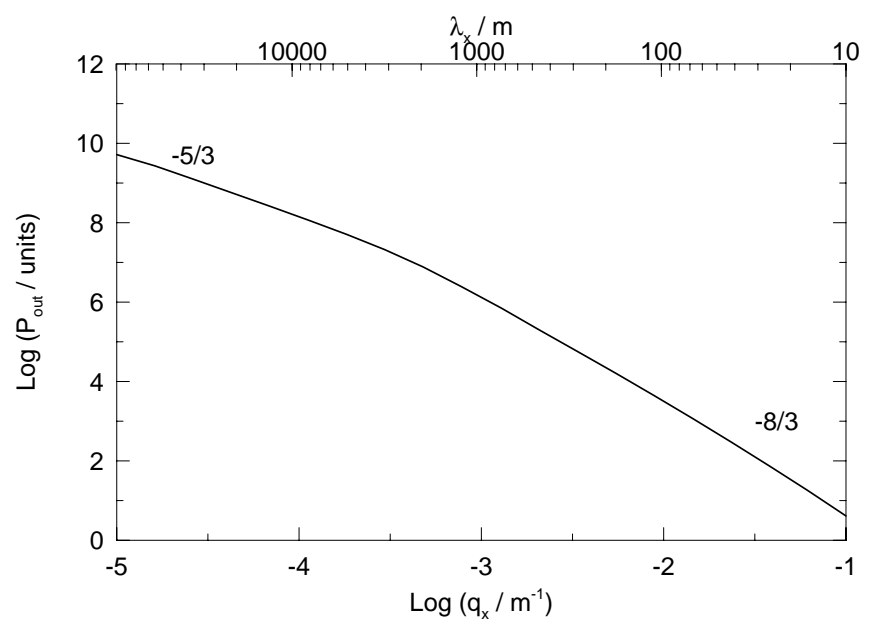

Fig. 5. $\log -\log$ plot to show how phase power changes as a function of size scale when the baseline is much longer than the thickness of the turbulent layer $(\Delta h=1 \mathrm{~km}$ in this case)

\subsection{The effect of elevation}

The results so far have dealt with antennas observing at an elevation of $90^{\circ}$. The effect of the elevation of the line of sight through the turbulent layer is complicated, and requires further elaboration of the model.

The coordinate system is now defined with the $z$-axis along the line of sight and the $x$-axis such that the $x-z$ plane contains the wind vector $\mathbf{w}$. The component of $\mathbf{w}$ in the $x$-direction is the projected windspeed $w_{x}$. The $z$-axis has elevation $\epsilon$ and azimuth in the horizontal plane $\theta$ with respect to $\mathbf{w}$. The baseline components $d_{x}$ and $d_{y}$ are in the $x$ - and $y$-directions, respectively, perpendicular to the line of sight; together they give the projected baseline.

The power spectrum of the wavefront delay is now given by

$$
\begin{aligned}
P_{\tau}\left(q_{x}, q_{y}\right)=A & \int_{-\infty}^{+\infty}\left\{\left(q_{x}+\Delta q_{x}\right)^{2}+\left(q_{y}+\Delta q_{y}\right)^{2}\right. \\
& \left.+\left(\Delta q_{z}\right)^{2}\right\}^{-\frac{11}{6}}(\Delta h)^{2} \operatorname{sinc}^{2}\left(\pi \Delta h q_{v}\right) \mathrm{d} q_{v}
\end{aligned}
$$

where $A$ is proportional to the strength of the turbulence,

$\Delta q_{x}=q_{v} \cos \epsilon \cos \theta$

$\Delta q_{y}=q_{v} \cos \epsilon \sin \theta$

$\Delta q_{z}=q_{v} \sin \epsilon$

and $q_{v}$ is the vertical component of each wavevector. Note that for $\epsilon=90^{\circ}$ Eq. (5) reduces to Eq. (4). The temporal power spectrum of the phase fluctuations at the output of the correlator is related to $P_{\tau}$ in the same way as before:

$P_{\text {out }}\left(q_{x}\right)=\left(2 \pi \nu_{\text {obs }}\right)^{2} \int_{-\infty}^{+\infty} 4 P_{\tau} \sin ^{2}\left\{\pi\left(d_{x} q_{x}+d_{y} q_{y}\right)\right\} \mathrm{d} q_{y},(9)$ 


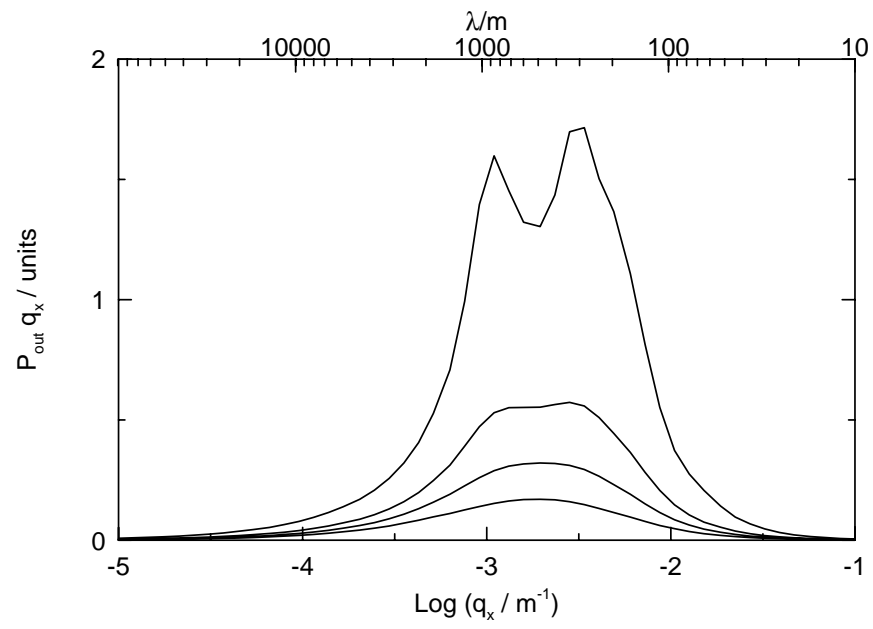

Fig. 6. Spectral density plots for elevations of $90^{\circ}, 45^{\circ}, 30^{\circ}$ and $15^{\circ}$, in order of increasing phase power. The turbulent layer is $100 \mathrm{~m}$ thick, the baseline has $d_{x}=0$ and $d_{y}=100 \mathrm{~m}$, and the lines of sight are inclined along the wind direction $(\theta=0$ in Eqs. (6) and (7)). The elevations correspond to airmasses of $1.0,1.4,2.0$ and 3.9

with $\nu=w_{x} q_{x}$.

Figure 6 shows examples of how the power spectrum obtained from a given turbulent layer is a function of the elevation. The curves are calculated by numerical integration of Eq. (5). The shape is a function of the relative values of $\Delta h, d_{x}, d_{y}, \epsilon$ and $\theta$. It can be seen that the phase power on small spatial scales (high $q_{x}$ ) is proportional to $\Delta h / \sin \epsilon$ as would be expected. It is also possible to show that the total phase power integrated over all timescales depends only on the length of the projected baseline $\left(d_{x}^{2}+d_{y}^{2}\right)^{1 / 2}$, the distance travelled through the turbulent layer $\Delta h / \sin \epsilon$ and the intensity of the turbulence $A$.

\subsection{Summary of the model}

A model has been developed to interpret the power spectrum of atmospheric turbulence measured by an interferometer. The distribution of phase power has a strong dependence on the orientation of the baseline with respect to the wind direction. The effects of the thickness of the turbulent layer and the elevation of the line of sight have also been demonstrated. The assumptions are that the geometrical optics approximation is valid over the scales of interest and that the turbulent field can be regarded as "frozen" Kolmogorov turbulence.

\section{Observations}

\subsection{The instrument}

The data presented here are from the atmospheric phase monitor at the Owens Valley Radio Observatory. This instrument comprises two $1.2 \mathrm{~m}$ off-axis antennas separated by an East-West baseline of $100 \mathrm{~m}$. The design is based on the system built by Masson et al. (1990), and is on loan from the Center for Astrophysics. The antennas are directed at a geosynchronous communications satellite in the South at an elevation of $43^{\circ}$ that emits an unmodulated tone at $11.7 \mathrm{GHz}$. The signals are down-converted and the phase difference between them is measured with a vector voltmeter and recorded every second. The phase difference varies with time as a result of turbulence in the atmosphere, drifts in the instrument response, and motions of the satellite along the line of sight. A dedicated phase monitor of this type provides a continuous record of the state of the atmosphere in a fixed direction on the sky, whereas measurements derived from bright astronomical sources are usually over only a limited period of time dictated by the observing schedule.

\subsection{Data processing}

The data are processed in 24 hours periods. There are several steps involved. First of all, phase wraps and $180^{\circ}$ phase jumps are removed. 12- and 24-hour sinusoids are then fitted to and subtracted from the data. This removes almost all of the satellite's radial motion. The data are then divided into segments of 4096 seconds (1 hour and 8 minutes). A straight line is fitted to and subtracted from each segment to remove drifts in the instrument and residual satellite motion, followed by a Fast Fourier Transform to generate 2048 complex values. The power spectrum is then given by the square of the amplitude of these values, and comprises 2048 measurements ranging in frequency from $0.5 \mathrm{~Hz}$ to $0.0 \mathrm{~Hz}$, spaced by $2.410^{-4} \mathrm{~Hz}$. Finally, the 20 or so power spectra generated for a 24 hour period are averaged together to produce the overall power spectrum for the day. The average rms phase, summed over all timescales up to $4096 \mathrm{~s}$, is also calculated.

The subtraction of a straight line from each segment changes the measured power spectrum. However the impact is minimal, since only the sine terms generated by the Fourier Transform are affected (the cosines are even functions with a first order moment of zero) and the power removed falls off as $q_{x}^{-2}$. In practice only the lowest two frequencies $\left(0.0 \mathrm{~Hz}\right.$ and $\left.2.410^{-4} \mathrm{~Hz}\right)$ are reduced significantly.

\subsection{The data}

The 4 data sets shown in Fig. 7 have been chosen to illustrate different conditions. These are discussed in the next section. Only a limited number of data sets have been 

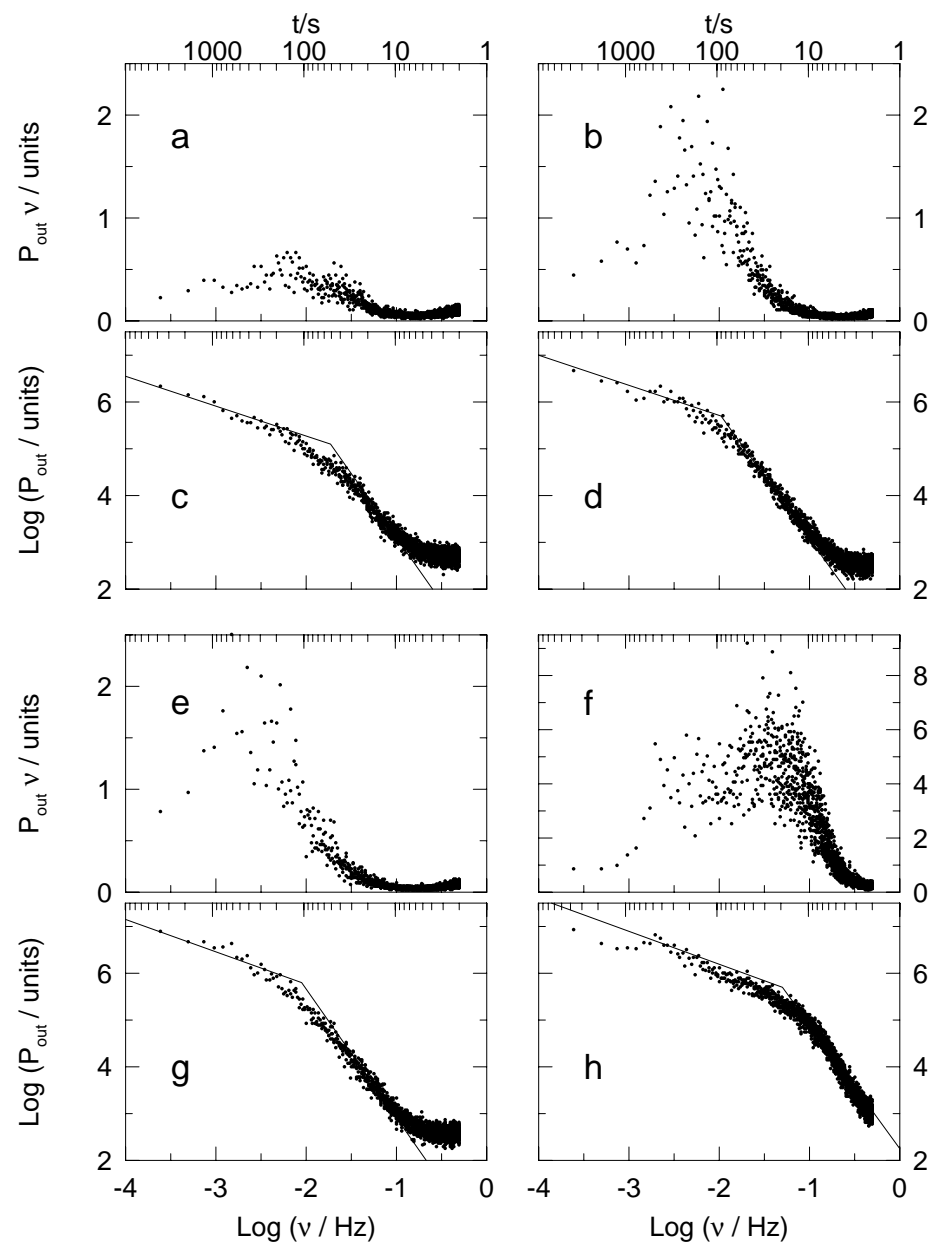

Fig. 7. a-h) Phase power plots and Log - Log plots for 4 days in the Owens Valley. a) and c) are for Oct. 25 1995, and illustrate a very calm atmosphere, setting upper limits on the instrumental contributions; b) and d) are for Feb. 41995 showing typical conditions; e) and g) are for Feb. 51995 which show that there can be substantial power on long timescales; f) and h) are for Jan. 121995 and show substantial power on short timescales, probably with two components. All but the last dataset have the same vertical scales. The lines on the Log - Log plots have gradients of $-8 / 3$ and $-2 / 3$, as predicted for a very thick layer of Kolmogorov turbulence, but no formal fitting has been made examined so far. Each of the four days of data is examined in turn and relevant issues are discussed.

\subsubsection{Oct. 251995}

The data of Figs. $7 \mathrm{a}$ and $\mathrm{c}$ are for one of the best days for which data is available. The rms phase on the $100 \mathrm{~m}$ baseline at $12 \mathrm{GHz}$, integrated over all timescales up to $4096 \mathrm{~s}$, is $1.6^{\circ}$ (equivalent to $110 \mu \mathrm{m}$ of path). The $\log -$ Log plot shows the signature expected from atmospheric phase noise; the straight lines have gradients of $-\frac{2}{3}$ and $-\frac{8}{3}$. There has been no attempt to make a formal fit to the data and the straight lines are shown for illustration only. The instrumental noise becomes apparent for frequencies exceeding $10^{-1} \mathrm{~Hz}$. The two peaks in the spectral density plot may be due to two distinct components of turbulence moving at different speeds in the atmosphere. The main purpose of showing this dataset is to set an upper limit on the contributions from instrumental noise and satellite motion.

\subsubsection{Feb. 41995}

The data of Figs. $7 \mathrm{~b}$ and d correspond to an integrated rms phase of $2.5^{\circ}$ at $12 \mathrm{GHz}$ (170 $\mu \mathrm{m}$ of path). The Log -
Log plot again shows the characteristic signature of the atmosphere and the contribution from instrumental noise for $\nu>10^{-1} \mathrm{~Hz}$. The data are more consistent with the models of Figs. $4 \mathrm{~d}$ and $\mathrm{f}$, where the wind is perpendicular to the baseline, than with $\mathrm{c}$ and $\mathrm{e}$, where the wind blows along the baseline direction. The Log - Log plot shows a more gradual transition between the two gradients than the data shown in Fig. $7 \mathrm{~h}$ where the wind is most likely along the baseline.

Figure 8 shows five model curves superimposed on the data. No formal fit has been made, but it is clear that the data are best fitted by $\Delta h$ in the range 100 to $1000 \mathrm{~m}$. The shape of the curve for $-2<\log \nu<-1$ is well-constrained by the data points and is fitted much better with the wind perpendicular to the baseline than along it. The projected windspeed required to map the spatial frequency scale of the model (wind perpendicular to baseline) to the temporal frequencies of the data is $(4.5 \pm 1) \mathrm{m} \mathrm{s}^{-1}$, or $9 \mathrm{mph}$. Since the elevation is $43^{\circ}$ in the direction of the wind, the actual windspeed needed to give a projected value of $9 \mathrm{mph}$ is $13 \mathrm{mph}$. The windspeed recorded at ground-level for that period was $\sim 5 \mathrm{mph}$. 


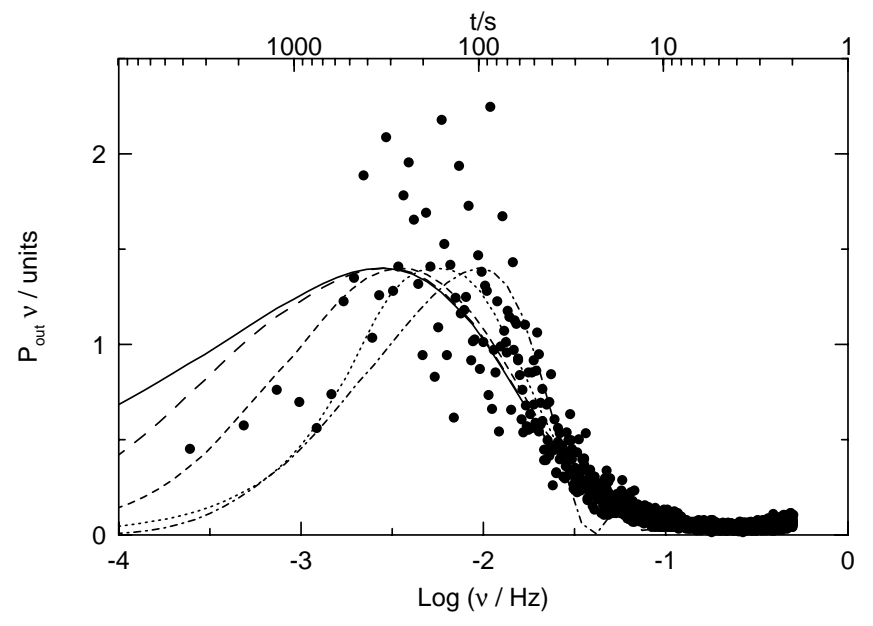

Fig. 8. Data for Feb. 41995 with five model curves superimposed. The solid, long dash, short dash and dotted lines have $\Delta h$ of $100 \mathrm{~km}, 5 \mathrm{~km}, 1 \mathrm{~km}$ and $100 \mathrm{~m}$, respectively, all with the wind perpendicular to a $100 \mathrm{~m}$ baseline. The dash-dot line has a $\Delta h$ of $1 \mathrm{~km}$, but with the wind blowing parallel to a $100 \mathrm{~m}$ baseline. All models are for an elevation of $45^{\circ}$, appropriate for the Owens Valley phase monitor

\subsubsection{Feb. 51995}

Panels e and $\mathrm{f}$ of Fig. 7 show data for the following day. The conditions appear to be similar to Feb. 41995 (the integrated rms phase is again $2.5^{\circ}$ at $12 \mathrm{GHz}$ ), except that the data are shifted to lower frequencies, indicative of a lower windspeed. The projected windspeed obtained is $2.5 \mathrm{~m} \mathrm{~s}^{-1}$ or $5 \mathrm{mph}$, requiring a $7 \mathrm{mph}$ wind perpendicular to the baseline, compared to the recorded value of $\sim 4 \mathrm{mph}$ at ground-level. There is still substantial phase power on timescales of $1000 \mathrm{~s}$ or more.

\subsubsection{Jan. 121995}

The data in panels $\mathrm{f}$ and $\mathrm{h}$ are clearly different in character from the preceding examples. There is substantial phase power on timescales as short as $10 \mathrm{~s}$. The data rise very rapidly from high $\nu$ and there is a more marked discontinuity in the gradients of the Log - Log plot. The integrated $\mathrm{rms}$ phase at $12 \mathrm{GHz}$ is $5.2^{\circ}$, corresponding to $360 \mu \mathrm{m}$ of path (note the different scaling on the spectral density plot).

The shape of the distribution in Fig. $7 \mathrm{f}$ can only be reconciled with the model if there are two components of turbulence present, moving at different speeds. One has a maximum centered on $\log \nu \simeq-1.3$ and dominates for $\log \nu>-2$; the second has a maximum at $\log \nu \simeq-2.3$, similar to the example in panel $\mathrm{b}$. To account for the sharp change in gradient of the Log - Log plot and the steep curve at $\log \nu \sim 1$ in the spectral density plot, the wind for the high frequency component must be approximately parallel to the baseline at $(25 \pm 5) \mathrm{m} \mathrm{s}^{-1}(50 \mathrm{mph})$. The second component has a projected windspeed of $\sim 9 \mathrm{mph}$, as for the example of Feb. 41995.

\section{Discussion}

The model provides a good framework for understanding and interpreting the data. In the datasets examined so far there are no obvious discrepancies with the model predictions. It would be straightforward to extend the model to different power-law relationships for the turbulence, but in the absence of obvious problems with the Kolmogorov model, and the lack of physical basis for another power law, this is not considered necessary at this stage. The data show that it is possible to have significant phase power on timescales as short as $10 \mathrm{~s}$ and as long as an hour.

\subsection{Turbulence and water vapor}

A turbulent velocity field can be generated in the atmosphere by a number of different processes, e.g. (1) convective activity from heating of the ground, (2) the passage of air past an obstacle, (3) instability at the interface of two layers with different wind vectors, and (4) the large scale motions associated with weather systems.

Each energy injection mechanism has a characteristic range of scales over which turbulence is generated. The Kolmogorov law assumes that the turbulence is in statistical equilibrium, with a constant energy input to replenish the energy dissipated on small scales. If turbulence is generated in a particular location (e.g. on the lee of a mountain) then at short distances downwind the turbulence will be lacking power on short scales since there has not been enough time for the energy to cascade down. Conversely, further downwind the large scale motions will have decayed without being replenished. Each case would be apparent as a deviation from the Kolmogorov behavior, the first as a decrement on short scales, the second as a decrement on large scales. Beyond the outer scale of the dominant mechanism there should also be a marked reduction in turbulent power. There is no clear evidence for any of these effects in the data sets studied so far. In Fig. 7 d, for example, there is no large deviation from the model at $t \sim 2000 \mathrm{~s}$; with a windspeed of $6.5 \mathrm{~m} \mathrm{~s}^{-1}$ this implies a lower limit to the outer scale of $\sim 10 \mathrm{~km}$. It is interesting to note that studies of the atmosphere at optical wavelengths, where the dominant contribution to phase fluctuations is the variation of the refractive index with temperature, suggest an outer scale size of order $5 \mathrm{~m}$ (e.g. Treuhaft et al. 1995; Coulman \& Vernin 1991). This is clearly not the case at radio wavelengths.

A mountain-top site is likely to be more complicated than a flat location. For example, experience with interferometry at submillimeter wavelengths on Mauna Kea 
has shown that there are times when there is substantial phase variation on 1-second timescales; similar periods are also apparent in data from the Plateau de Bure in France (Bremer 1995).

The presence of a turbulent velocity field is not enough in itself to generate inhomogeneity in the distribution of water vapor. There must also be some initial density contrast in the distribution of water vapor within the turbulent zone; a uniform distribution cannot be mixed, and will not give rise to variations in the refractivity under the influence of turbulence. The total power of the phase fluctuations therefore depends on the density contrast in the water vapor that would be present in the turbulent region in the absence of turbulence. For example, convection mixes water-rich air from low altitude with drier regions higher up. A higher column density of water vapor does not directly imply stronger phase fluctuations. This may explain the weak dependence of radio seeing with altitude found by Masson (1993).

The model may be used to estimate the fractional contribution to the water vapor column along a single line of sight from the varying component. For typical conditions in the Owens Valley (5-minute rms phase at $12 \mathrm{GHz}$ of $2.5^{\circ}$, and $5 \mathrm{~mm}$ of precipitable water vapor) the varying component constitutes $\sim 5 \%$ of the total water vapor column.

\subsection{Frozen turbulence}

It is possible to estimate the validity of the assumption of frozen turbulence and to show the effect that non-frozen flows will have on the temporal power spectrum.

Consider an element of turbulence with size-scale $l$. The lifetime of this feature is of order $l / v_{l}$ (see Tatarskii 1961, Chap. 2), where $v_{l}$ is the turbulent velocity of the element. Therefore the timescale over which the feature retains a coherent identity is $t_{\mathrm{coh}} \sim l / v_{l}$. In this time the feature is blown a distance $w l / v_{l}$, where $w$ is the windspeed. If $w l / v_{l}>l$, i.e. $w>v_{l}$ then the feature passes through a given line of sight relatively unchanged, and the assumption of frozen turbulence is a good approximation for this scale.

From simple dimensional arguments (Tatarskii 1961, Chap. 2), $v_{l} \propto l^{1 / 3}$, so that the velocity of the turbulent motion is highest on the largest scales, and this is where the frozen turbulence assumption will break down first. The wind is usually the result of motions on the scale of hundreds of kilometers. It is reasonable to assume that when turbulence is produced by the wind blowing past an obstacle or by the wind shear between layers, the turbulent motions do not have speeds exceeding $w$. If the turbulence is dominated by convection and the systematic "background" windspeed is very low, then it is possible that the velocity of the turbulence exceeds the windspeed and large structures evolve faster than the blow-by time. In this case there will be an apparent deficit of phase power on long timescales. Frozen turbulence should therefore be a good approximation whenever the systematic windspeed exceeds the speed of convective motions.

\subsection{The geometrical optics approximation}

This approximation, as noted in Sect. 2, assumes that the wavefront delay having passed through an inhomogeneous medium is given by the integral of the refractivity variations along the line of sight. The effects of diffraction are ignored. Tatarskii (1961, Chap. 6) shows that diffraction becomes important on size scales $l$ for which $l \lesssim \sqrt{\lambda_{\text {obs }} h}$, where $\lambda_{\text {obs }}$ is the observing wavelength and $h$ is the distance between the inhomogeneity and the observer.

The phase monitor observes at a wavelength of $25 \mathrm{~mm}$, so that the approximation is valid only for scales exceeding $\sim 5 \mathrm{~m}$. Reference to Fig. 4 shows that there is actually very little phase power from scales less than $5 \mathrm{~m}$. Diffraction results in some of this power being spread to larger spatial scales, so in this case the approximation has very little impact on the power spectrum. It will become more important for observations at lower frequencies and shorter baselines.

\subsection{Turbulence in the Owens Valley}

The Owens Valley (Fig. 9) runs North-South, is approximately $8 \mathrm{~km}$ wide, and the floor is at an elevation of $1200 \mathrm{~m}$. To the West, the mountains of the Sierra Nevada rise abruptly to over $4000 \mathrm{~m}$; to the East the White Mountain range rises to $\sim 3000 \mathrm{~m}$. The wind direction at the observatory on the valley floor is almost always North-South, but the prevailing wind direction for elevations exceeding $4000 \mathrm{~m}$ is approximately East-West. The obvious sources of turbulence are convective activity from the valley floor, eddies generated by the passage of air over the Sierra Nevada, and shearing between the volume of air in the valley and the air moving over the mountains. An analysis of the rms phase measured by the phase monitor over 5-minute intervals over a period of several months shows clearly that the phase tends to be worst during the middle of the afternoon. This suggests that convective activity inside the valley, blown in a North-South direction perpendicular to the baseline of the phase monitor, is a dominant contributor. This is consistent with the findings of the previous section. Turbulence generated by the fast air blowing over the Sierra Nevada from West to East is the likely cause of the high frequency component in Figs. $8 \mathrm{f}$ and h. This is shown schematically in Fig. 9.

\subsection{Extrapolating phase monitor data}

The main aim of this paper is to demonstrate that a simple model with few assumptions can explain the basic features of the phase monitor data taken in the Owens Valley. This model can then be used to extrapolate the phase monitor data to different baselines and elevations to assess the 


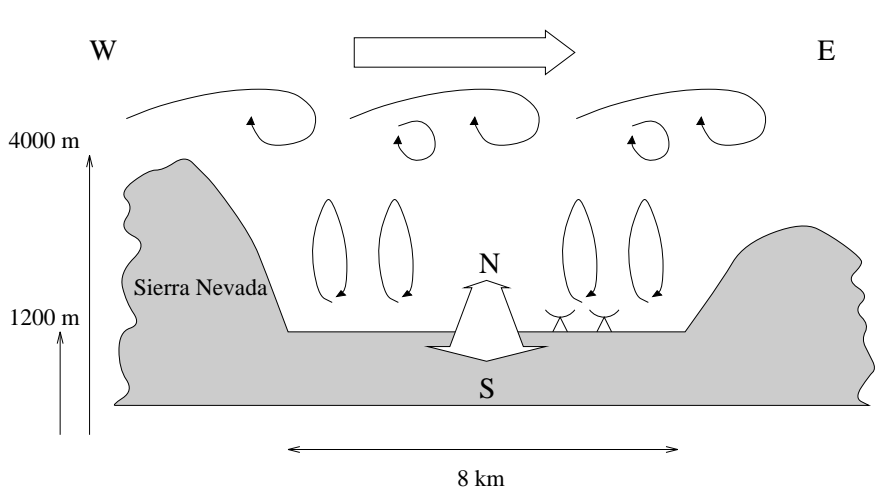

Fig. 9. Schematic illustration of the Owens Valley. Convective turbulence in the valley is blown North-South. High-altitude turbulence is generated by the Sierra Nevada mountains and is blown predominantly to the West. The phase monitor is located on the valley floor with an East-West baseline

impact of atmospheric fluctuations on astronomical data measured with interferometers.

The model can be fitted to a measured temporal power spectrum to estimate the wind speed (horizontal shift of the curve) and direction (shape of the curve for the high frequencies), turbulent intensity (vertical scaling) and the thickness of the turbulent layer (width of the curve). An example is given in Fig. 8. With these quantities the model can then be used to predict the power spectrum for an arbitrary baseline, elevation and observing frequency.

\subsection{Advantages of the temporal power spectrum approach}

The primary motivation for investigating the temporal power spectrum here is to use the data to evaluate the effect of phase calibration and correction schemes using water vapor radiometry. A knowledge of the timescales of fluctuations is clearly needed to do this, and the traditional structure function provides no such information. The spectral density distribution is also much more sensitive to the presence of multiple components of turbulence (e.g. Fig. 7h) which can be distinguished on the basis of windspeed.

The measured spectral density distributions also show that there can be substantial phase power on timescales longer than $1000 \mathrm{~s}$ for a baseline of only $100 \mathrm{~m}$. When measuring the structure function, it is vital that the phase is monitored over a sufficiently long period of time. The longer the baseline, the longer the time required. Figure $4 \mathrm{~d}$ has power on spatial scales 100 times the baseline length and the necessary sampling period can be prohibitive, particularly if the windspeed is low. If the period is too short, the power law index of the structure function will be underestimated.

A discussion of phase calibration procedures based on the temporal power spectrum of phase fluctuations, and the implications for water vapor radiometer schemes that will attempt to correct the fluctuations, is the subject of a companion paper (Lay 1997). It will also be instructive to study a much larger sample of data to look for cases where the model is inadequate, and to investigate diurnal variations.

\section{Summary}

The Kolmogorov model for turbulence in the atmosphere is used to predict the power spectrum of phase fluctuations measured by a radio interferometer. Spectral density plots, where the phase fluctuation power is proportional to the area under the curve, are shown to be very useful for appreciating the relevant timescales, and there are straightforward scalings to different baseline length and airmass. The model is extended to include the thickness of the turbulent layer and the orientation of the baseline with respect to the wind direction.

The data examined so far are in broad agreement with the model. There can be significant phase variations on timescales as long as one hour. There may also be more than one component of turbulence contributing to the phase fluctuations. There is no clear evidence for an outer scale of turbulence on scales less than $\sim 10 \mathrm{~km}$, in contrast to optical observations.

Acknowledgements. The author would like to thank Rachel Akeson, John Carlstrom, Peter Papadopoulos and David Woody for many useful comments, and acknowledges a Robert A. Millikan Fellowship from Caltech.

\section{References}

Armstrong J.W., Sramek R.A., 1982, Radio Sci. 17, 1579

Bremer M., 1995, The Phase Project: Observations of Quasars, IRAM Working Report No. 238

Coulman C.E., Vernin J., 1991, Appl. Opt. 30, 118

Holdaway M.A., 1992, Possible phase calibration schemes for the MMA, MMA Memo 84

Holdaway M.A., Owen F.N., 1995, A test of fast switching phase calibration with the VLA at $22 \mathrm{GHz}$, MMA Memo 126

Ishiguro M., Kanazawa T., Kasuga T., 1990, Monitoring of Atmospheric Phase Fluctuations using Geostationary Satellite Signals. In: Baldwin J.E., \& Wang Shouguan (eds.) Radio Astronomical Seeing. Pergamon, Oxford, p. 60

Lay O.P., 1997, A\&AS 122, 547

Masson C.R., Williams J.D., Oberlander D., Hernstein J., 1990, Submillimeter Array Technical Memorandum No. 30

Masson C.R., 1993, Seeing. In: Robertson J.G., Tango W.J. (eds.) Very High Angular Resolution Imaging. Kluwer, Dordrecht

Masson C.R., 1994, Atmospheric Effects and Calibrations. In: Ishiguro M. \& Welch W.J. (eds.) Astronomy with Millimeter and Submillimeter Wave Interferometry, ASP Conf. Ser. 59, 87

Olmi L., Downes D., 1992, A\&A 262, 634 
Sramek R.A., 1990, Atmospheric Phase Stability at the VLA. In: Baldwin J.E., \& Wang Shouguan (eds.) Radio Astronomical Seeing. Pergamon, Oxford, p. 21

Tatarskii V.I., 1961, Wave Propagation in a Turbulent Medium. Dover: New York

Tatarskii V.I., 1971, The Effects of the Turbulent Atmosphere on Wave Propagation, Israel Program for Scientific Translations: Jerusalem

Tatarskii V.I., Ishimaru A., Zavorotny V.U., 1992, Wave Propagation in Random Media (Scintillation), SPIE, Washington

Thompson A.R., Moran J.M., Swenson G., 1986, Interferometry and Synthesis in Radio Astronomy.
Wiley-Interscience

Treuhaft R.N., Lanyi G.E., 1987, Radio Sci. 22, 251

Treuhaft R.N., Lowe S.T., Bester M., Danchi W.C., Townes C.H., 1995, ApJ 453, 522

Welch Wm. J., 1994, The Berkeley-Illinois-MarylandAssociation Array. In: Ishiguro M. \& Welch W.J. (eds.) Astronomy with Millimeter and Submillimeter Wave Interferometry, ASP Conf. Ser. 59, 74

Wright M.C.H., Welch Wm.J., 1990, Interferometer Measurements of Atmospheric Phase Noise. In: Radio Astronomical Seeing, Baldwin J.E. \& Wang Shouguan (eds.). Pergamon, Oxford, p. 71 